\title{
A period map for generalized deformations
}

\author{
Domenico Fiorenza and Marco Manetti
}

\begin{abstract}
For every compact Kähler manifold we give a canonical extension of Griffith's period map to generalized deformations, intended as solutions of Maurer-Cartan equation in the algebra of polyvector fields. Our construction involves the notion of Cartan homotopy and a canonical $L_{\infty}$-structure on mapping cones of morphisms of differential graded Lie algebras.
\end{abstract}

Mathematics Subject Classification (2000). 14D07, 17B70, 13D10.

Keywords. Differential graded Lie algebras, symmetric coalgebras, $L_{\infty}$-algebras, functors of Artin rings, Kähler manifolds, period map.

\section{Introduction}

Let $X$ be a compact Kähler manifold and denote by $H^{*}(X, \mathbb{C})$ the graded vector space of its de Rham cohomology. The goal of this article is to define a natural transformation

$$
\Phi: \operatorname{Def}_{X} \rightarrow \operatorname{Aut}_{H *(X, \mathbb{C})}
$$

from infinitesimal deformations of $X$ to automorphisms of $H^{*}(X, \mathbb{C})$. More precisely, for every local Artinian $\mathbb{C}$-algebra $A$ and every deformation of $X \operatorname{over} \operatorname{Spec}(A)$ we define in a functorial way a canonical morphism of schemes

$$
\operatorname{Spec}(A) \rightarrow \operatorname{GL}\left(H^{*}(X, \mathbb{C})\right)=\prod_{n} \operatorname{GL}\left(H^{n}(X, \mathbb{C})\right)
$$

Our construction will be carried out by using the interplay of Cartan homotopies and $L_{\infty}$-morphisms and is compatible with classical constructions of the theory of infinitesimal variations of Hodge structures [11], [21]. In particular:

(1) Via the natural isomorphism $H_{X} \simeq \bigoplus_{p, q} H^{q}\left(\Omega_{X}^{p}\right)$ induced by Dolbeault's theorem and the $\partial \bar{\partial}$-lemma, the differential of $\Phi$,

$$
d \Phi: H^{1}\left(T_{X}\right) \rightarrow \operatorname{Hom}^{0}\left(H^{*}(X, \mathbb{C}), H^{*}(X, \mathbb{C})\right)
$$

is identified with the contraction operator: $d \Phi(\xi)=\boldsymbol{i}_{\xi}$ where $\left.\boldsymbol{i}_{\xi}(\omega)=\xi\right\lrcorner \omega$. 
(2) The contraction

$$
i: H^{2}\left(T_{X}\right) \rightarrow \operatorname{Hom}^{1}\left(H^{*}(X, \mathbb{C}), H^{*}(X, \mathbb{C})\right)
$$

is a morphism of obstruction theories. In particular, since $\operatorname{Aut}_{H *}(X, \mathbb{C})$ is smooth, every obstruction to deformation of $X$ is contained in the kernel of $\boldsymbol{i}$.

(3) For every $m$ let $H^{*}\left(F^{m}\right) \subseteq H^{*}(X, \mathbb{C})$ be the subspace of cohomology classes of closed $(p, q)$-forms, with $p \geq m$. Then the composition of $\Phi$ with the natural projection

$$
\begin{aligned}
\operatorname{GL}\left(H^{*}(X, \mathbb{C})\right) & \rightarrow \operatorname{Grass}\left(H^{*}(X, \mathbb{C})\right)=\prod_{n} \operatorname{Grass}\left(H^{n}(X, \mathbb{C})\right), \\
f & \mapsto f\left(H^{*}\left(F^{m}\right)\right),
\end{aligned}
$$

is the classical period map.

We will define and study the morphism $\Phi$ using the framework of $L_{\infty}$-algebras. It is however useful to give also a more geometric definition in the following way.

Denote by $A_{X}=\bigoplus_{i} A_{X}^{i}$ the space of complex valued differential forms on $X$, by $d=\partial+\bar{\partial}: A_{X}^{i} \rightarrow A_{X}^{i+1}$ the de Rham differential and by $\partial A_{X} \subseteq A_{X}$ the subspace of $\partial$-exact forms. A small variation of the almost complex structure is determined by a form $\xi \in A_{X}^{0,1}\left(T_{X}\right)$ : according to Newlander-Niremberg theorem, the integrability condition of $\xi$ is equivalent to $\left(d+\boldsymbol{l}_{\xi}\right)^{2}=0$, where $\boldsymbol{l}_{\xi}: A_{X}^{i} \rightarrow A_{X}^{i+1}$ is the holomorphic Lie derivative, defined by the formula

$$
\left.\left.\boldsymbol{l}_{\xi}(\omega)=\partial(\xi\lrcorner \omega\right)+\xi\right\lrcorner \partial \omega .
$$

Notice that $\boldsymbol{l}_{\xi}(\operatorname{ker} \partial) \subseteq \partial A_{X}$.

Assume therefore $\left(d+l_{\xi}\right)^{2}=0$. According to $\partial \bar{\partial}$-lemma, the complex $\left(\partial A_{X}, d\right)$ is acyclic so that if $\xi$ is sufficiently small, the complex $\left(\partial A_{X}, d+\boldsymbol{l}_{\xi}\right)$ is still acyclic.

In order to define the automorphism $\Phi_{\xi}: H^{*}(X, \mathbb{C}) \rightarrow H^{*}(X, \mathbb{C})$, let $[\omega] \in$ $H^{*}(X, \mathbb{C})$ and choose a $d$-closed form $\omega_{0} \in A_{X}$ representing $[\omega]$ and such that $\partial \omega_{0}=0$. Since

$$
\left.\left(d+\boldsymbol{l}_{\xi}\right) \omega_{0}=\partial(\xi\lrcorner \omega_{0}\right) \in \partial A_{X} \quad \text { and } \quad\left(d+\boldsymbol{l}_{\xi}\right)^{2} \omega_{0}=0,
$$

there exists $\beta \in A_{X}$ such that $\left(d+\boldsymbol{l}_{\xi}\right) \omega_{0}=\left(d+\boldsymbol{l}_{\xi}\right) \partial \beta$. If $\boldsymbol{i}_{\xi}$ is the contraction, then it is not difficult to prove that $d\left(e^{i} \xi\left(\omega_{0}-\partial \beta\right)\right)=0$, and the cohomology class of $e^{\boldsymbol{i}_{\xi}}\left(\omega_{0}-\partial \beta\right)$ does not depend on the choice of $\beta$ and $\omega_{0}$, allowing to define $\Phi_{\xi}([\omega])$ as the cohomology class of $e^{\boldsymbol{i}_{\xi}}\left(\omega_{0}-\partial \beta\right)$.

Equivalently, for every $d$-closed form $\omega \in A_{X}$ and every small variation of the complex structure $\xi$ we have

$$
\Phi_{\xi}([\omega])=\left[e^{\boldsymbol{i}_{\xi}}(\omega-d \gamma-\partial \beta)\right] \text {, where } \partial \omega=\partial d \gamma, \boldsymbol{l}_{\xi}(\omega-d \gamma)=\left(d+\boldsymbol{l}_{\xi}\right) \partial \beta .
$$

Moreover, as direct consequence of the $L_{\infty}$-approach, we will see that $\Phi_{\xi}$ is invariant under the gauge action, where two integrable small variation of the almost 
complex structure $\xi_{1}, \xi_{2}$ are gauge equivalent if and only if they give isomorphic deformations of $X$.

Our construction generalizes in a completely straightforward way to generalized deformations of $X$, defined as the solutions, up to gauge equivalence, of the MaurerCartan equation in the differential graded Lie algebra

$$
\operatorname{Poly}_{X}=\bigoplus_{i} \text { Poly }_{X}^{i}, \quad \operatorname{Poly}_{X}^{i}=\underset{b-a=i-1}{\bigoplus_{X}} A_{X}^{0, b}\left(\bigwedge^{a} T_{X}\right),
$$

endowed with the opposite of Dolbeault differential and the Schouthen-Nijenhuys bracket.

Putting together all these facts, at the end we get for every $m$ a commutative diagram of morphism of functors of Artin rings

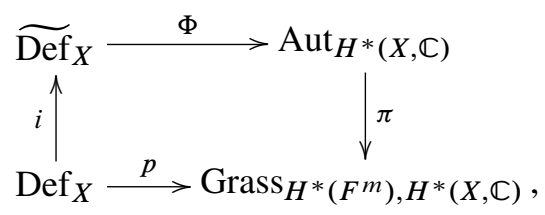

where $\widetilde{\operatorname{Def}_{X}}$ is the functor of generalized deformations of $X, i$ is the natural inclusion, $\operatorname{Grass}_{H^{*}}\left(F^{m}\right), H^{*}(X, \mathbb{C})$ is the Grassmann functor with base point $H^{*}\left(F^{m}\right), \pi$ is the smooth morphism defined as $\pi(f)=f\left(H^{*}\left(F^{m}\right)\right)$ and $p$ is the classical $m^{\text {th }}$ period map.

In view of this result is natural to candidate the composition $\pi \Phi$ as period map for generalized deformations.

Example. Our definition is compatible with yet existing notion of period map for generalized deformations of Calabi-Yau manifolds used in some mirror symmetry constructions [3].

In fact, if $X$ is a Calabi-Yau manifold with volume element $\Omega$, then by the TianTodorov Lemma, every generalized deformation over $\operatorname{Spec}(A)$ is represented by an element $\xi \in \operatorname{Poly}_{X}^{1} \otimes \mathfrak{m}_{A}$ such that

$$
\left.D \xi+\frac{1}{2}[\xi, \xi]=0, \quad \partial(\xi\lrcorner \Omega\right)=0 .
$$

Under these assumptions our recipe gives

$$
\Phi_{\xi}([\Omega])=\left[e^{i_{\xi}}(\Omega)\right]
$$

and therefore we recover the construction of [2].

Keywords and general notation. We assume that the reader is familiar with the notion and main properties of differential graded Lie algebras and $L_{\infty}$-algebras (we refer to [8], [14], [15], [16], [19] as introduction to such structures); however the basic 
definitions are recalled in this article in order to fix notation and terminology. For the whole article, $\mathbb{K}$ is a field of characteristic 0 ; every vector space is intended over $\mathbb{K}$. Art is the category of local Artinian $\mathbb{K}$-algebras with residue field $\mathbb{K}$. For $A \in$ Art we denote by $\mathfrak{m}_{A}$ the maximal ideal of $A$. By abuse of notation, if $F:$ Art $\rightarrow$ Set is a functor, we write $\xi \in F$ to mean $\xi \in F(A)$ for some fixed $A \in$ Art.

Acknowledgments. We thank the referee for useful comments and for suggesting a possible extension of the constructions presented in this article to generalized Kähler manifolds [12].

\section{Deformation functors associated with DGLA morphisms}

We recall from [20] that to any morphism $\chi: L \rightarrow M$ of differential graded Lie algebras over a field $\mathbb{K}$ of characteristic 0 are naturally associated two functors $\mathrm{MC}_{\chi}, \operatorname{Def}_{\chi}:$ Art $\rightarrow$ Set of Artin rings in the following way:

$$
\begin{aligned}
& \operatorname{MC}_{\chi}(A)=\left\{\left(x, e^{a}\right) \in\left(L^{1} \otimes \mathfrak{m}_{A}\right) \times \exp \left(M^{0} \otimes \mathfrak{m}_{A}\right) \mid d x+\frac{1}{2}[x, x]\right.=0, \\
&\left.e^{a} * \chi(x)=0\right\}, \\
& \operatorname{Def}_{\chi}(A)=\frac{\operatorname{MC}_{\chi}(A)}{\text { gauge equivalence }},
\end{aligned}
$$

where two solutions of the Maurer-Cartan equation are gauge equivalent if they belong to the same orbit of the gauge action

$$
\left(\exp \left(L^{0} \otimes \mathfrak{m}_{A}\right) \times \exp \left(d M^{-1} \otimes \mathfrak{m}_{A}\right)\right) \times \operatorname{MC}_{\chi}(A) \stackrel{*}{\longrightarrow} \operatorname{MC}_{\chi}(A)
$$

given by the formula

$$
\left(e^{l}, e^{d m}\right) *\left(x, e^{a}\right)=\left(e^{l} * x, e^{d m} e^{a} e^{-\chi(l)}\right)=\left(e^{l} * x, e^{d m \bullet a \bullet(-\chi(l))}\right) .
$$

The in the rightmost term in the above formula is the Baker-Campbell-Hausdorff multiplication; namely $e^{x} e^{y}=e^{x \bullet y}$. Note that if $L=0$ and the differential on $M$ is trivial, then

$$
\operatorname{Def}_{\chi}(A)=\exp \left(M^{0} \otimes \mathfrak{m}_{A}\right)
$$

It has been shown in [6] that the suspended cone of $\chi$, i.e., the differential complex $\left(C_{\chi}, \mu_{1}\right)$ given by the graded vector space

$$
C_{\chi}=\bigoplus_{i} C_{\chi}^{i}, \quad C_{\chi}^{i}=L^{i} \oplus M^{i-1},
$$

endowed with the differential

$$
\mu_{1}(l, m)=(d l, \chi(l)-d m), \quad l \in L, m \in M,
$$


carries a natural compatible $L_{\infty}$-algebra structure, which we shall denote $\widetilde{C}(\chi)$, such that the associated deformation functor $\operatorname{Def}_{\widetilde{C}(\chi)}$ is naturally isomorphic to $\operatorname{Def}_{\chi}$. More precisely, the map $(l, m) \mapsto\left(l, e^{m}\right)$ induces a natural isomorphism $\mathrm{MC}_{\tilde{C}(\chi)} \stackrel{\sim}{\rightarrow} \mathrm{MC}_{\chi}$, and homotopy equivalence on $\mathrm{MC}_{\widetilde{C}(\chi)}$ is identified with gauge equivalence on $\mathrm{MC}_{\chi}$.

The higher brackets

$$
\mu_{n}: \stackrel{n}{\wedge} C_{\chi} \rightarrow C_{\chi}[2-n], \quad n \geq 2,
$$

defining the $L_{\infty}$-algebra structure $\widetilde{C}(\chi)$ have been explicitly described in [6]. Namely, one has

$$
\mu_{2}\left(\left(l_{1}, m_{1}\right) \wedge\left(l_{2}, m_{2}\right)\right)=\left(\left[l_{1}, l_{2}\right], \frac{1}{2}\left[m_{1}, \chi\left(l_{2}\right)\right]+\frac{(-1)^{\operatorname{deg}\left(l_{1}\right)}}{2}\left[\chi\left(l_{1}\right), m_{2}\right]\right)
$$

and for $n \geq 3$

$$
\begin{aligned}
& \mu_{n}\left(\left(l_{1}, m_{1}\right) \wedge \cdots \wedge\left(l_{n}, m_{n}\right)\right) \\
& \quad= \pm \frac{B_{n-1}}{(n-1) !} \sum_{\sigma \in S_{n}} \varepsilon(\sigma)\left[m_{\sigma(1)},\left[\ldots,\left[m_{\sigma(n-1)}, \chi\left(l_{\sigma(n)}\right)\right] \ldots\right]\right] .
\end{aligned}
$$

Here the $B_{n}$ 's are the Bernoulli numbers, $\varepsilon$ is the Koszul sign, and we refer to [6] for the exact determination of the overall \pm sign in the above formulas (it will not be needed in the present article). Note that the projection on the first factor $\pi_{1}: \widetilde{C}(\chi) \rightarrow L$ is a linear $L_{\infty}$-morphism.

By the functoriality of $\chi \mapsto \widetilde{C}(\chi)$, if

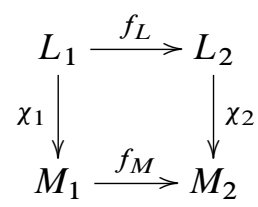

is a commutative diagram of morphisms of differential graded Lie algebras, then $\left(x, e^{a}\right) \mapsto\left(f_{L}(x), e^{f_{M}(a)}\right)$ is a natural transformation of Maurer-Cartan functors inducing a natural transformation

$$
\operatorname{Def}_{\chi_{1}} \rightarrow \operatorname{Def}_{\chi_{2}} .
$$

Moreover, if $f_{L}$ and $f_{M}$ are quasi-isomorphisms, then $\operatorname{Def}_{\chi_{1}} \stackrel{\sim}{\rightarrow} \operatorname{Def}_{\chi_{2}}$ is an isomorphism.

\section{An example from Kähler geometry}

Let $X$ be a compact Kähler manifold. Consider the DGLA Hom* $\left(A_{X}, A_{X}\right)$ of graded endomorphisms of the de Rham complex and their subDGLAs

$$
\begin{aligned}
L & =\left\{f \in \operatorname{Hom}^{*}\left(A_{X}, A_{X}\right) \mid f(\operatorname{ker} \partial) \subseteq \partial A_{X}\right\}, \\
M & =\left\{f \in \operatorname{Hom}^{*}\left(A_{X}, A_{X}\right) \mid f(\operatorname{ker} \partial) \subseteq \operatorname{ker} \partial \text { and } f\left(\partial A_{X}\right) \subseteq \partial A_{X}\right\} .
\end{aligned}
$$


Then we have a commutative diagram of morphisms of DGLAs, where the vertical arrows are the inclusions

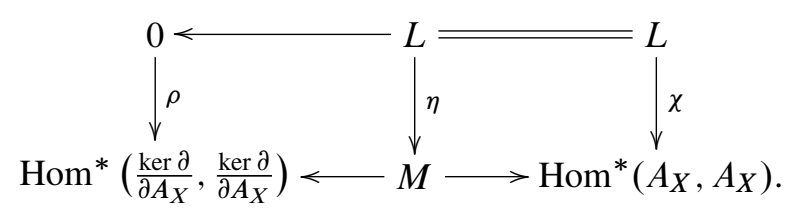

By the $\partial \bar{\partial}$-lemma, we have quasi-isomorphisms

$$
\left(A_{X}, d\right) \leftarrow(\operatorname{ker} \partial, d) \rightarrow\left(\frac{\operatorname{ker} \partial}{\partial A_{X}}, 0\right) \cong\left(H^{*}(X, \mathbb{C}), 0\right) .
$$

Hence the horizontal arrows in the above commutative diagram are quasi-isomorphisms and we get isomorphisms of deformation functors

$$
\operatorname{Def}_{\chi} \simeq \operatorname{Def}_{\eta} \simeq \operatorname{Def}_{\rho}=\operatorname{Aut}_{H *}(X, \mathbb{C})
$$

The isomorphism $\psi: \operatorname{Def}_{\chi} \simeq \operatorname{Aut}_{H^{*}(X, \mathbb{C})}$ is explicitly described as follows: Given a Maurer-Cartan element $\left(\alpha, e^{a}\right) \in \mathrm{MC}_{\chi}$ and a cohomology class $[\omega] \in H^{*}(X, \mathbb{C})$,

$$
\psi_{a}([\omega])=\left[e^{\tilde{a}} \omega_{0}-\partial \beta_{0}\right]
$$

where $\left(\tilde{\alpha}, e^{\tilde{a}}\right) \in \mathrm{MC}_{\eta} \subseteq \mathrm{MC}_{\chi}$ is gauge-equivalent to $\left(\alpha, e^{a}\right)$, the differential form $\omega_{0}$ is a $\partial$-closed representative for the cohomology class $[\omega]$, and $\beta_{0} \in A_{X}$ is such that $d\left(e^{\tilde{a}} \omega_{0}-\partial \beta_{0}\right)=0$. The cohomology class $\left[e^{\tilde{a}} \omega_{0}-\partial \beta_{0}\right]$ is independent of the choices of $\left(\tilde{\alpha}, e^{\tilde{a}}\right), \omega_{0}$ and $\beta_{0}$. Since $e^{\tilde{a}}$ is an automorphism of $\partial A_{X}$, we can write

$$
\psi_{a}([\omega])=\left[e^{\tilde{a}}\left(\omega_{0}-\partial \beta_{0}\right)\right]
$$

for any $\left(\tilde{\alpha}, e^{\tilde{a}}\right)$ and $\omega_{0}$ as above and any $\beta \in A_{X}$ such that $d e^{\tilde{a}}\left(\omega_{0}-\partial \beta\right)=0$.

Remark 2.1. As $\chi: L \hookrightarrow \operatorname{Hom}^{*}\left(A_{X}, A_{X}\right)$ is injective, the projection on the second factor $C_{\chi} \rightarrow \operatorname{Hom}^{*-1}\left(A_{X}, A_{X}\right)$ induces an identification $H^{*}\left(C_{\chi}\right) \simeq H^{*-1}(\operatorname{coker} \chi)$ and so in particular

$$
H^{1}\left(C_{\chi}\right) \stackrel{\sim}{\rightarrow} H^{0}\left(\operatorname{Hom}^{*}\left(\operatorname{ker} \partial, A_{X} / \partial A_{X}\right)\right)=\operatorname{Hom}^{0}\left(H^{*}(\operatorname{ker} \partial), H^{*}\left(A_{X} / \partial A_{X}\right)\right) \text {. }
$$

Hence the differential of $\psi$ is naturally identified with the linear isomorphism

$$
\operatorname{Hom}^{0}\left(H^{*}(\operatorname{ker} \partial), H^{*}\left(A_{X} / \partial A_{X}\right)\right) \simeq \operatorname{Hom}^{0}\left(H^{*}(X, \mathbb{C}) ; H^{*}(X, \mathbb{C})\right),
$$

induced by the $\partial \bar{\partial}$-lemma and the de Rham isomorphism.

For later use, we give a more explicit description of the map $\psi$ by writing a map $\widetilde{\psi}: \mathrm{MC}_{\chi} \rightarrow \operatorname{Aut}_{H *(X, \mathbb{C})}$ inducing it. To define the map $\widetilde{\psi}$ we need a few preliminary remarks. 
Lemma 2.2. If $\left(\alpha, e^{a}\right) \in \mathrm{MC}_{\chi}$, then in the associative algebra $\operatorname{Hom}^{*}\left(A_{X}, A_{X}\right)$ we have the equality

$$
e^{-a} d e^{a}=d+\alpha
$$

Proof. By the definition of the gauge action in the DGLA $\operatorname{Hom}^{*}\left(A_{X}, A_{X}\right)$, one has for every $x \in \operatorname{Hom}^{0}\left(A_{X}, A_{X}\right), y \in \operatorname{Hom}^{1}\left(A_{X}, A_{X}\right)$ the formula

$$
e^{x} * y=e^{x}(d+y) e^{-x}-d .
$$

In particular, $e^{-a} d e^{a}=d+e^{-a} * 0$.

Corollary 2.3. If $\left(\alpha, e^{a}\right) \in \mathrm{MC}_{\chi}$, then the graded subspaces

$$
e^{a}(\operatorname{ker} \partial), \quad e^{a}\left(\partial A_{X}\right)
$$

are subcomplexes of $\left(A_{X}, d\right)$. Moreover, the map

$$
e^{a}:\left(\frac{\mathrm{ker} \partial}{\partial A_{X}}, 0\right) \rightarrow\left(\frac{e^{a}(\mathrm{ker} \partial)}{e^{a}\left(\partial A_{X}\right)}, 0\right)
$$

is an isomorphism of complexes and the natural maps

$$
\left(A_{X}, d\right) \leftarrow\left(e^{a}(\operatorname{ker} \partial), d\right) \rightarrow\left(\frac{e^{a}(\operatorname{ker} \partial)}{e^{a}\left(\partial A_{X}\right)}, 0\right)
$$

are quasi-isomorphisms.

Proof. Both $\left(\partial A_{X}, d\right)$ and $(\operatorname{ker} \partial, d)$ are subcomplexes of $\left(A_{X}, d\right)$. Because $d e^{a}(v)=e^{a}(d v+\alpha(v))$ with $\alpha(\operatorname{ker} \partial) \subseteq \partial A_{X}$, we have $d e^{a}\left(\partial A_{X}\right) \subseteq e^{a}\left(\partial A_{X}\right)$ and $d e^{a}(\operatorname{ker} \partial) \subseteq e^{a}(\operatorname{ker} \partial)$. The induced differential on the quotient space $\frac{e^{a}(\operatorname{ker} \partial)}{e^{a}\left(\partial A_{X}\right)}$ is trivial since $d(\operatorname{ker} \partial) \subseteq \partial A_{X}$ by the $\partial \bar{\partial}$-lemma. Again by the $\partial \bar{\partial}$-lemma the complex $\left(\partial A_{X}, d\right)$ is acyclic and therefore the morphisms of complexes

$$
\left(A_{X}, d\right) \leftarrow(\operatorname{ker} \partial, d) \rightarrow\left(\frac{\operatorname{ker} \partial}{\partial A_{X}}, 0\right)
$$

are quasi-isomorphisms. Since every infinitesimal perturbation of an acyclic complex is still acyclic, the complex $\left(e^{a}\left(\partial A_{X}\right), d\right)$ is acyclic and so the morphisms of complexes

$$
\left(A_{X}, d\right) \leftarrow\left(e^{a}(\operatorname{ker} \partial), d\right) \rightarrow\left(\frac{e^{a}(\operatorname{ker} \partial)}{e^{a}\left(\partial A_{X}\right)}, 0\right)
$$

are quasi-isomorphisms.

Definition 2.4. The isomorphism

$$
\widetilde{\psi}_{a}: H^{*}(X, \mathbb{C}) \rightarrow H^{*}(X, \mathbb{C})
$$


associated to a Maurer-Cartan element $\left(\alpha, e^{a}\right)$ via the natural map $\mathrm{MC}_{\chi} \rightarrow \operatorname{Def}_{\rho} \simeq$ $\operatorname{Aut}^{0}\left(H^{*}(X ; \mathbb{C})\right.$ is obtained by the de Rham isomorphism $H^{*}(X, \mathbb{C})=H^{*}\left(A_{X}, d\right)$ and the chain of quasi-isomorphisms

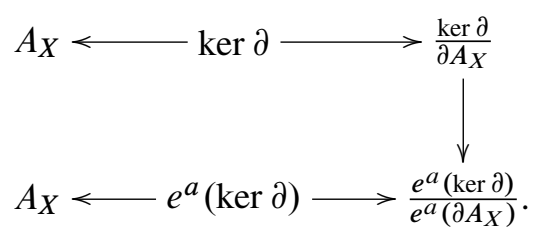

More explicitly,

$$
\widetilde{\psi}_{a}([\omega])=\left[e^{a}\left(\omega_{0}-\partial \beta\right)\right]
$$

for any $\partial$-closed representative $\omega_{0}$ of the cohomology class $[\omega]$ and any $\beta \in A_{X}$ such that $d e^{a}\left(\omega_{0}-\partial \beta\right)=0$.

Proposition 2.5. The natural transformation $\tilde{\psi}: \mathrm{MC}_{\chi} \rightarrow \operatorname{Aut}_{H^{*}(X, \mathbb{C})}$ is gauge invariant and therefore factors to $\psi: \operatorname{Def}_{\chi} \rightarrow \operatorname{Aut}_{H^{*}}(X, \mathbb{C})$.

Proof. To show that $\tilde{\psi}_{a \bullet(-l)}=\widetilde{\psi}_{a}$, note that, since $l($ ker $\partial) \subseteq \partial A_{X}$, we have $e^{-l}(\operatorname{ker} \partial)=\operatorname{ker} \partial, e^{-l}\left(\partial A_{X}\right)=\partial A_{X}$, and

$$
e^{-l}:\left(\frac{\operatorname{ker} \partial}{\partial A_{X}}, 0\right) \rightarrow\left(\frac{\operatorname{ker} \partial}{\partial A_{X}}, 0\right)
$$

is the identity. To prove that $\widetilde{\psi}_{d m \bullet a}=\widetilde{\psi}_{a}$, notice that we have a commutative diagram of morphisms of complexes

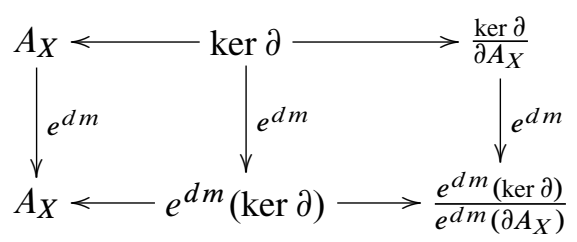

which, since $d m$ is homotopy equivalent to zero, induces the commutative diagram of isomorphisms

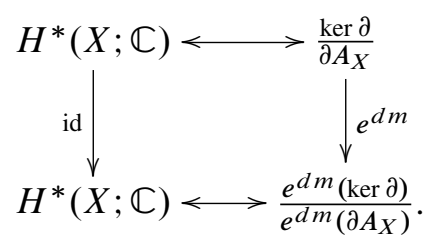

Finally, gauge invariance of $\widetilde{\psi}$, together with the explicit formulae for $\widetilde{\psi}([\omega])$ and $\psi([\omega])$ written above immediately imply that $\widetilde{\psi}$ induces $\psi$. 


\section{Morphisms of deformation functors associated to Cartan homotopies}

In this section we formalize, under the notion of Cartan homotopy, a set of standard identities that often arise in algebra and geometry [4], Appendix B, and show how to any Cartan homotopy can be canonically associated a natural transformation of deformation functors.

Let $L$ and $M$ be two differential graded Lie algebras. For a given linear map $i \in \operatorname{Hom}^{-1}(L, M)$, let $\boldsymbol{l}: L \rightarrow M$ be the map defined as

$$
a \mapsto \boldsymbol{l}_{a}=d \boldsymbol{i}_{a}+\boldsymbol{i}_{d a} .
$$

Definition 3.1. The map $\boldsymbol{i}$ is called a Cartan homotopy for $\boldsymbol{l}$ if

$$
\boldsymbol{i}_{[a, b]}=\left[\boldsymbol{i}_{a}, \boldsymbol{l}_{b}\right], \quad\left[\boldsymbol{i}_{a}, \boldsymbol{i}_{b}\right]=0 .
$$

for every $a, b \in L$.

It is straightforward to show that the condition $\boldsymbol{i}_{[a, b]}=\left[\boldsymbol{i}_{a}, \boldsymbol{l}_{b}\right]$ implies that $\boldsymbol{l}$ is a morphism of differential graded Lie algebras.

Example 3.2. The name Cartan homotopy has a clear origin in differential geometry. Namely, let $M$ be a differential manifold, $\mathcal{X}(M)$ be the Lie algebra of vector fields on $M$, and $\operatorname{End}^{*}\left(\Omega^{*}(M)\right)$ be the Lie algebra of endomorphisms of the de Rham algebra of $M$. The Lie algebra $\mathcal{X}(M)$ can be seen as a DGLA concentrated in degree zero, and the graded Lie algebra $\operatorname{End}^{*}\left(\Omega^{*}(M)\right)$ has a degree one differential given by $\left[d_{\mathrm{dR}},-\right]$, where $d_{\mathrm{dR}}$ is the de Rham differential. Then the contraction

$$
i: \mathcal{X}(M) \rightarrow \operatorname{End}^{*}\left(\Omega^{*}(M)\right)[-1]
$$

is a Cartan homotopy and its differential is the Lie derivative

$$
[d, i]=\mathscr{L}: \mathcal{X}(M) \rightarrow \operatorname{End}^{*}\left(\Omega^{*}(M)\right) .
$$

In fact, by classical Cartan's homotopy formulas [1], Section 2.4, for any two vector fields $X$ and $Y$ on $M$, we have

(1) $\mathscr{L}_{X}=d_{\mathrm{dR}} \boldsymbol{i}_{X}+\boldsymbol{i}_{X} d_{\mathrm{dR}}=\left[d_{\mathrm{dR}}, \boldsymbol{i}_{X}\right]$,

(2) $\boldsymbol{i}_{[X, Y]}=\mathscr{L}_{X} \boldsymbol{i}_{Y}-\boldsymbol{i}_{Y} \mathscr{L}_{X}=\left[\mathscr{L}_{X}, \boldsymbol{i}_{Y}\right]=\left[\boldsymbol{i}_{X}, \mathscr{L}_{Y}\right]$,

(3) $\left[i_{X}, i_{Y}\right]=0$.

Note that the first Cartan formula above actually states that $[d, i]=\mathscr{L}$. Indeed $\mathcal{X}(M)$ is concentrated in degree zero and then its differential is trivial.

Remark 3.3. The composition of a Cartan homotopy with a morphism of DGLAs is a Cartan homotopy. If $\boldsymbol{i}: L \rightarrow M[-1]$ is a Cartan homotopy and $\Omega$ is a differential graded-commutative algebra, then its natural extension

$$
i \otimes \mathrm{id}: L \otimes \Omega \rightarrow(M \otimes \Omega)[-1], \quad a \otimes \omega \mapsto \boldsymbol{i}_{a} \otimes \omega,
$$

is a Cartan homotopy. 
Remark 3.4. By definition, $\boldsymbol{l}$ is the differential of $\boldsymbol{i}$ in the complex $\operatorname{Hom}^{*}(L, M)$ and so $\boldsymbol{i}$ is a homotopy between $\boldsymbol{l}$ and the trivial map. Then the map $\boldsymbol{l}: L \rightarrow M$ is a null-homotopic morphism of DGLAs and

$$
\boldsymbol{i}: L \rightarrow(\operatorname{coker} \boldsymbol{l})[-1], \quad \boldsymbol{i}: \operatorname{ker} \boldsymbol{l} \rightarrow M[-1]
$$

are morphisms of differential graded vector spaces.

Proposition 3.5. Let $\boldsymbol{l}: L \rightarrow M$ be a DGLA morphism, and let $\boldsymbol{i}: L \rightarrow M[-1]$ be a Cartan homotopy for $\boldsymbol{l}$. Then the linear map $\tilde{\boldsymbol{i}}: L \rightarrow \widetilde{\boldsymbol{C}}(\boldsymbol{l})$ given by $\tilde{\boldsymbol{i}}(a)=\left(a, \boldsymbol{i}_{a}\right)$ is an $L_{\infty}$-morphism. In particular, the map $a \mapsto\left(a, e^{\boldsymbol{i}_{a}}\right)$ induces a natural transformation of Maurer-Cartan functors $\mathrm{MC}_{L} \rightarrow \mathrm{MC}_{l}$, and consequently a natural transformation of deformation functors $\operatorname{Def}_{L} \rightarrow \operatorname{Def}_{l}$.

Proof. By the explicit expression for the higher brackets

$$
\mu_{n}: \stackrel{n}{\wedge} C_{\chi} \rightarrow C_{\chi}[2-n], \quad n \geq 2,
$$

defining the $L_{\infty}$-algebra structure $\widetilde{C}(\chi)$, it is straightforward to check that $\tilde{\boldsymbol{i}}$ is a morphism of complexes commuting with every bracket. Indeed, $\tilde{\boldsymbol{i}}(d a)=\mu_{1}(\tilde{\boldsymbol{i}}(a))$ is the identity $\boldsymbol{i}_{d a}=(-d) \boldsymbol{i}_{a}+\boldsymbol{l}_{a}$; the identity $\tilde{\boldsymbol{i}}([a, b])=\mu_{2}(\tilde{\boldsymbol{i}}(a) \wedge \tilde{\boldsymbol{i}}(b))$ is

$$
\boldsymbol{i}_{[a, b]}=\frac{1}{2}\left[\boldsymbol{i}_{a}, \boldsymbol{l}_{b}\right]+\frac{(-1)^{\operatorname{deg}(a)}}{2}\left[\boldsymbol{l}_{a}, \boldsymbol{i}_{b}\right]=\left[\boldsymbol{i}_{a}, \boldsymbol{l}_{b}\right],
$$

and $\mu_{n}\left(\tilde{\boldsymbol{i}}\left(x_{1}\right) \wedge \cdots \wedge \tilde{\boldsymbol{i}}\left(x_{n}\right)\right)=0$ for any $x_{1}, x_{2}, \ldots, x_{n}$ and any $n \geq 3$, since

$$
\left[\boldsymbol{i}_{a},\left[\boldsymbol{i}_{b}, \boldsymbol{l}_{c}\right]=\left[\boldsymbol{i}_{a}, \boldsymbol{i}_{[b, c]}\right]=0\right.
$$

for any $a, b, c$.

Since the $L_{\infty}$-morphism $\tilde{\boldsymbol{i}}$ is linear, the map $l \mapsto\left(l, \boldsymbol{i}_{l}\right)$ is a morphism of MaurerCartan functors $\mathrm{MC}_{L} \rightarrow \mathrm{MC}_{\widetilde{C}(\boldsymbol{l})}$. To conclude the proof, compose this morphism with the isomorphism $\mathrm{MC}_{\widetilde{C}(l)} \stackrel{\sim}{\rightarrow} \operatorname{MC}_{\boldsymbol{l}}$ given by $(l, m) \mapsto\left(l, e^{m}\right)$.

Corollary 3.6. Let $\boldsymbol{i}: N \rightarrow M[-1]$ be a Cartan homotopy for $\boldsymbol{l}: N \rightarrow M$, let $L$ be a subDGLA of $M$ such that $\boldsymbol{l}(N) \subseteq L$, and let $\chi: L \hookrightarrow M$ be the inclusion. Then the linear map

$$
\Phi: N \rightarrow \widetilde{C}(\chi), \quad \Phi(a)=\left(\boldsymbol{l}_{a}, \boldsymbol{i}_{a}\right),
$$

is a linear $L_{\infty}$-morphism. In particular, the map $a \mapsto\left(\boldsymbol{l}_{a}, e^{\boldsymbol{i}_{a}}\right)$ induces a natural transformation of Maurer-Cartan functors $\mathrm{MC}_{N} \rightarrow \mathrm{MC}_{\chi}$, and consequently a natural transformation of deformation functors $\operatorname{Def}_{N} \rightarrow \operatorname{Def}_{\chi}$.

Proof. We have a commutative diagram of differential graded Lie algebras

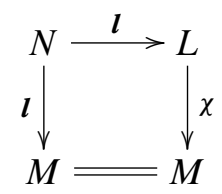


inducing an $L_{\infty}$-morphism $\widetilde{C}(\boldsymbol{l}) \rightarrow \widetilde{C}(\chi)$. Composing this morphism with the $L_{\infty^{-}}$ morphism $\tilde{\boldsymbol{i}}: N \rightarrow \widetilde{C}(\boldsymbol{l})$ given by Proposition 3.5 , one gets the $L_{\infty}$-morphism $\Phi$.

\section{Polyvector fields and generalized periods}

The notion of Cartan homotopy generalizes immediately to sheaves of DGLAs. In this section we give another example of Cartan homotopy, which will be used later.

Let $X$ be a complex manifold and denote by

- $T_{X, \mathbb{C}}=T_{X}^{1,0} \oplus T_{X}^{0,1}$ the complexified differential tangent bundle,

- $T_{X} \simeq T_{X}^{1,0}$ the holomorphic tangent bundle,

- $\mathcal{A}_{X}^{p, q}$ the sheaf of differentiable $(p, q)$-forms and by $\mathcal{A}_{X}^{p, q}(E)$ the sheaf of $(p, q)$ forms with values in a holomorphic vector bundle $E$,

- $A_{X}^{p, q}$ and $A_{X}^{p, q}(E)$ the vector spaces of global sections of $\mathcal{A}_{X}^{p, q}$ and $\mathcal{A}_{X}^{p, q}(E)$, respectively.

The direct sum

$$
\mathcal{A}_{X}=\bigoplus_{i} \mathcal{A}_{X}^{i}, \quad \text { where } \mathcal{A}_{X}^{i}=\bigoplus_{p+q=i} \mathcal{A}_{X}^{p, q},
$$

endowed with the wedge product $\wedge$, is a sheaf of graded algebras; we denote by $\mathscr{H}_{o m}^{a, b}\left(\mathcal{A}_{X}, \mathcal{A}_{X}\right)$ the sheaf of its $\mathbb{C}$-linear endomorphisms of $\mathcal{A}_{X}$ of bidegree $(a, b)$. Notice that $\bar{\partial}$ and $\bar{\partial}$ are global sections of $\mathscr{H o m}^{1,0}\left(\mathcal{A}_{X}, \mathcal{A}_{X}\right)$ and $\mathscr{H o m}^{0,1}\left(\mathcal{A}_{X}, \mathcal{A}_{X}\right)$, respectively. The direct sum $\mathscr{H} o m^{*}\left(\mathcal{A}_{X}, \mathcal{A}_{X}\right)=\bigoplus_{k} \bigoplus_{a+b=k} \mathscr{H} o m^{a, b}\left(\mathcal{A}_{X}, \mathcal{A}_{X}\right)$ is a sheaf of graded associative algebras, and so a sheaf of differential graded Lie algebras with the natural bracket

$$
[f, g]=f g-(-1)^{\operatorname{deg}(f) \operatorname{deg}(g)} g f
$$

and differential $[d,-]=[\partial+\bar{\partial},-]$.

For any integer $(a, b)$ with $a \leq 0$ and $b \geq 0$, let $\mathcal{G e r s t}_{X}^{a, b}$ be the sheaf

$$
\operatorname{Gerst}_{X}^{a, b}=\mathcal{A}_{X}^{0, b}\left(\wedge^{-a} T_{X}\right)
$$

The direct sum $\mathcal{E}_{\text {erst }}^{*}{ }_{X}=\bigoplus_{k} \bigoplus_{a+b=k} \mathcal{E}_{e r s t}^{a, b}$ is a sheaf of differential Gerstenhaber algebras, with the wedge product

$$
\wedge: \operatorname{Eerst}_{X}^{a_{1}, b_{1}} \otimes \operatorname{Eerst}_{X}^{a_{2}, b_{2}} \rightarrow \operatorname{Eerst}_{X}^{a_{1}+a_{2}, b_{1}+b_{2}}
$$

as graded commutative product, the degree 1 differential

$$
\bar{\partial}: \operatorname{Eerst}_{X}^{a, b} \rightarrow \operatorname{Eerst}_{X}^{a, b+1}
$$


defined in local coordinates by the formula

$$
\bar{\partial}\left(\phi \frac{\partial}{\partial z_{I}}\right)=\bar{\partial}(\phi) \frac{\partial}{\partial z_{I}}, \quad \phi \in \mathcal{A}_{X}^{0, *},
$$

and the degree 1 bracket

$$
[\cdot, \cdot]_{\mathrm{G}}: \operatorname{Eerst}_{X}^{a_{1}, b_{1}} \otimes \operatorname{Gerst}_{X}^{a_{2}, b_{2}} \rightarrow \operatorname{Eerst}_{X}^{a_{1}+a_{2}+1, b_{1}+b_{2}}
$$

defined in local coordinates by the formula

$$
\left[f d \bar{z}_{I} \frac{\partial}{\partial z_{H}}, g d \bar{z}_{J} \frac{\partial}{\partial z_{K}}\right]_{\mathrm{G}}=d \bar{z}_{I} \wedge d \bar{z}_{J}\left[f \frac{\partial}{\partial z_{H}}, g \frac{\partial}{\partial z_{K}}\right]_{\mathrm{SN}} .
$$

Here $[\cdot, \cdot]_{\text {SN }}$ denotes the Schouten-Nijenhuis bracket on $\mathcal{A}_{X}^{0}\left(\wedge^{*} T_{X}\right)$, i.e., the odd graded Lie bracket obtained by extending the usual Lie bracket on $\mathcal{A}_{X}^{0}\left(T_{X}\right)$ by imposing

$$
[\xi, f]_{\mathrm{SN}}=\xi(f), \quad \xi \in \mathcal{A}^{0}\left(T_{X}\right), \quad f \in \mathcal{A}_{X}^{0}
$$

and the odd graded Poisson identity

$$
\left[\xi, \eta_{1} \wedge \eta_{2}\right]_{\mathrm{SN}}=\left[\xi, \eta_{1}\right]_{\mathrm{SN}} \wedge \eta_{2}+(-1)^{\operatorname{deg}(\xi)\left(\operatorname{deg}\left(\eta_{1}\right)-1\right)} \eta_{1} \wedge\left[\xi, \eta_{2}\right]_{\mathrm{SN}} .
$$

The contraction of differential forms with vector fields is used to define an injective morphisms of sheaves of bigraded vector spaces: the contraction map

$$
\left.\boldsymbol{i}: \mathcal{E}_{\text {erst }}^{a, b} \rightarrow \operatorname{Hom}^{a, b}\left(\mathcal{A}_{X}, \mathcal{A}_{X}\right), \quad \xi \mapsto \boldsymbol{i}_{\xi}, \boldsymbol{i}_{\xi}(\omega)=\xi\right\lrcorner \omega .
$$

The contraction map is actually a morphism of sheaves of bigraded associative algebras:

$$
\boldsymbol{i}_{\xi \wedge \eta}=\boldsymbol{i}_{\xi} \boldsymbol{i}_{\eta}
$$

In particular, since $\left(\mathscr{G} e r s t_{X}^{*}, \wedge\right)$ is a graded commutative algebra, we obtain that

$$
\left[\boldsymbol{i}_{\xi}, \boldsymbol{i}_{\eta}\right]=0, \quad \text { for all } \xi, \eta \in \mathcal{E}_{\text {erst }}^{*} .
$$

Note that iterated contractions give a symmetric map

$$
\boldsymbol{i}^{(n)}: \bigodot^{n} \mathcal{E}_{e r s t_{X}^{*}} \rightarrow \mathcal{H o m}^{*}\left(\mathcal{A}_{X}, \mathcal{A}_{X}\right), \quad \xi_{1} \odot \xi_{2} \odot \cdots \odot \xi_{n} \mapsto \boldsymbol{i}_{\xi_{1}} \boldsymbol{i}_{\xi_{2}} \ldots \boldsymbol{i}_{\xi_{n}} .
$$

Since $\mathcal{E}_{e r s t}^{*}{ }_{X}$ is a sheaf of differential graded Gerstenhaber algebras, its desuspension

$$
\left.\operatorname{Poly}_{X}^{*}=\operatorname{Eerst}_{[-1}\right]_{X}^{*}
$$

is a sheaf of differential graded Lie algebras. Note that, due to the shift, the differential $D$ in $\mathcal{P o l y}_{X}^{*}$ is $-\bar{\partial}$, i.e., in local coordinates

$$
D: \operatorname{Poly}_{X}^{k} \rightarrow \operatorname{Poly}_{X}^{k+1}
$$


is given by the formula

$$
D\left(\phi \frac{\partial}{\partial z_{I}}\right)=-\bar{\partial}(\phi) \frac{\partial}{\partial z_{I}}, \quad \phi \in \mathcal{A}_{X}^{0, *}
$$

The contraction map $i: \mathscr{G e r s t}_{X}^{*} \rightarrow \mathcal{H o m}^{*}\left(\mathcal{A}_{X}, \mathcal{A}_{X}\right)$ can be seen as a linear map

$$
i: \operatorname{Poly}_{X}^{*} \rightarrow \operatorname{Hom}^{*}\left(\mathcal{A}_{X}, \mathcal{A}_{X}\right)[-1]
$$

More general, via the decalage isomorphism, the iterated contraction is a graded antisymmetric map

$$
i^{(n)}: \bigwedge^{n} \operatorname{Poly}_{X}^{*} \rightarrow \mathcal{H o m}^{*}\left(\mathcal{A}_{X}, \mathcal{A}_{X}\right)[-n]
$$

Lemma 4.1. In the notation above, for every $\xi, \eta \in \mathcal{P o l y}_{X}^{*}$ we have

$$
\boldsymbol{i}_{D \xi}=-\left[\bar{\partial}, \boldsymbol{i}_{\xi}\right], \quad \boldsymbol{i}_{[\xi, \eta]}=\left[\boldsymbol{i}_{\xi},\left[\partial, \boldsymbol{i}_{\eta}\right]\right], \quad\left[\boldsymbol{i}_{\xi}, \boldsymbol{i}_{\eta}\right]=0 .
$$

Proof. The third equation has been proved above, and the first equation is completely straightforward: it just expresses the Leibniz rule for $\bar{\partial}$. To prove the second equation, let

$$
\Phi(\xi, \eta)=\boldsymbol{i}_{[\xi, \eta]}-\left[\boldsymbol{i}_{\xi},\left[\partial, \boldsymbol{i}_{\eta}\right]\right]
$$

Then, using $\boldsymbol{i}_{\xi \wedge \eta}=\boldsymbol{i}_{\xi} \boldsymbol{i}_{\eta}$, the (shifted) odd Poisson identity $\left[\xi, \eta_{1} \wedge \eta_{2}\right]=\left[\xi, \eta_{1}\right] \wedge$ $\eta_{2}+(-1)^{(\operatorname{deg}(\xi)-1) \operatorname{deg}\left(\eta_{1}\right)} \eta_{1} \wedge\left[\xi, \eta_{2}\right]$ and the third equation $\left[\boldsymbol{i}_{\xi}, \boldsymbol{i}_{\eta}\right]=0$, one finds

$$
\Phi\left(\xi, \eta_{1} \wedge \eta_{2}\right)=\Phi\left(\xi, \eta_{1}\right) \boldsymbol{i}_{\eta_{2}}+(-1)^{(\operatorname{deg}(\xi)-1) \operatorname{deg}\left(\eta_{1}\right)} \boldsymbol{i}_{\eta_{1}} \Phi\left(\xi, \eta_{2}\right)
$$

and

$$
\Phi\left(\xi_{1} \wedge \xi_{2}, \eta\right)=\boldsymbol{i}_{\xi_{1}} \Phi\left(\xi_{2}, \eta\right)+(-1)^{\left(\operatorname{deg}\left(\xi_{2}\right)(\operatorname{deg}(\eta)-1)\right.} \Phi\left(\xi_{1}, \eta\right) \boldsymbol{i}_{\eta_{2}}
$$

Therefore, to prove $\Phi(\xi, \eta)=0$ for any $\xi, \eta$ one just needs to prove $\Phi(\xi, \eta)=0$ for $\xi, \eta \in \mathcal{A}_{X}^{0} \cup\left\{d \bar{z}_{i}\right\} \cup\left\{\partial / \partial z_{j}\right\}$, where $z_{1}, \ldots, z_{n}$ are local holomorphic coordinates. This is straightforward and is left to the reader; see also Lemma 7 of [18] and Lemma 7.21 of [19].

Corollary 4.2. The contraction map $i: \operatorname{Poly}_{X}^{*} \rightarrow \mathscr{H o m}^{*}\left(\mathcal{A}_{X}, \mathcal{A}_{X}\right)[-1]$ is a Cartan homotopy and the induced morphism $\boldsymbol{l}$ of sheaves of differential graded Lie algebras is the holomorphic Lie derivative

$$
\begin{aligned}
\boldsymbol{l}: \operatorname{Poly}_{X}^{*} & \rightarrow \operatorname{Hom}^{*}\left(\mathcal{A}_{X}, \mathcal{A}_{X}\right), \\
\xi & \left.\left.\mapsto \boldsymbol{l}_{\xi}=\left[\partial, \boldsymbol{i}_{\xi}\right], \boldsymbol{l}_{\xi}(\omega)=\partial(\xi\lrcorner \omega\right)+(-1)^{\operatorname{deg}(\xi)} \xi\right\lrcorner \partial \omega .
\end{aligned}
$$

Moreover, $\boldsymbol{l}$ is an injective morphism of sheaves. 
Proof. As in Section 3, let $\boldsymbol{l}_{\xi}=\left[d, \boldsymbol{i}_{\xi}\right]+\boldsymbol{i}_{D \xi}$. Since $\boldsymbol{i}_{D \xi}=-\left[\bar{\partial}, \boldsymbol{i}_{\xi}\right]$, we find $\boldsymbol{l}_{\xi}=\left[\partial, \boldsymbol{i}_{\xi}\right]$. The identity $\boldsymbol{i}_{[\xi, \eta]}=\left[\boldsymbol{i}_{\xi},\left[\partial, \boldsymbol{i}_{\eta}\right]\right]$ then $\operatorname{reads} \boldsymbol{i}_{[\xi, \eta]}=\left[\boldsymbol{i}_{\xi}, \boldsymbol{l}_{\eta}\right]$ and this together with $\left[\boldsymbol{i}_{\xi}, \boldsymbol{i}_{\eta}\right]=0$ tells us that the contraction map $\boldsymbol{i}$ is a Cartan homotopy. Consequently, the holomorphic Lie derivative $\boldsymbol{l}_{\xi}=\left[\partial, \boldsymbol{i}_{\xi}\right]$ is the induced morphism of sheaves of differential graded Lie algebras. Injectivity of $\boldsymbol{l}$ is easily checked in local coordinates.

Corollary 4.2, applied to global sections, shows that the contraction map

$$
i: \operatorname{Poly}_{X}=\bigoplus_{i, j} A^{0, i}\left(\bigwedge^{j} T_{X}\right) \rightarrow \operatorname{Hom}^{*}\left(\mathcal{A}_{X}, \mathcal{A}_{X}\right)[-1]
$$

is a Cartan homotopy, as well as its restriction to the Kodaira-Spencer DGLA $\mathrm{KS}_{X}=\bigoplus_{i} A^{0, i}\left(T_{X}\right)$.

Remark 4.3. The composition of the inclusion $\mathrm{KS}_{X} \hookrightarrow$ Poly $_{X}$ with the iterated contraction $\boldsymbol{i}^{(n)}: \bigwedge^{n} \operatorname{Poly}_{X} \rightarrow \operatorname{Hom}^{*}\left(\mathcal{A}_{X}, \mathcal{A}_{X}\right)[-n]$ induces in cohomology a graded antisymmetric map $\bigwedge^{n} H^{*}\left(\mathrm{KS}_{X}\right) \rightarrow \operatorname{Hom}^{*}\left(H^{*}(X, \mathbb{C}), H^{*}(X, \mathbb{C})\right)[-n]$. In particular, from the isomorphism of graded vector spaces $H^{1}\left(\mathrm{KS}_{X}^{*}\right) \simeq H^{1}\left(T_{X}\right)[-1]$ and the decalage isomorphism, the iterated contraction gives a symmetric morphism

$$
i^{(n)}: \bigodot^{n} H^{1}\left(T_{X}\right) \rightarrow \operatorname{Hom}^{0}\left(H^{*}(X, \mathbb{C}), H^{*}(X, \mathbb{C})\right) .
$$

It is well known [10] and easy to prove that the image of $\boldsymbol{i}^{(n)}$ consists of self-adjoint operators with respect the cup product on $H^{*}(X, \mathbb{C})$.

Under the identification $H^{*}(X, \mathbb{C})=\bigoplus_{p, q} H^{q}\left(X, \Omega_{X}^{p}\right)$, when $\operatorname{dim} X=n$ the morphism $\boldsymbol{i}^{(n)}$ reduces to the Yukawa coupling

$$
i^{(n)}: \bigodot^{n} H^{1}\left(T_{X}\right) \rightarrow \bigodot^{2} H^{n}\left(X, \mathcal{O}_{X}\right) .
$$

Now, as in Section 2, consider the DGLA

$$
L=\left\{f \in \operatorname{Hom}^{*}\left(A_{X}, A_{X}\right) \mid f(\operatorname{ker} \partial) \subseteq \partial A_{X}\right\},
$$

and let $\chi: L \hookrightarrow \operatorname{Hom}^{*}\left(A_{X}, A_{X}\right)$ be the inclusion. Since $\boldsymbol{l}\left(\operatorname{Poly}_{X}\right) \subseteq L$ by Corollary 3.6, we have a natural transformation of deformation functors $\operatorname{Def}_{\mathrm{Poly}_{X}} \rightarrow \operatorname{Def}_{\chi}$ induced, at the Maurer-Cartan level, by the map $\xi \mapsto\left(l_{\xi}, e^{i_{\xi}}\right)$.

The functor $\operatorname{Def}_{\text {Poly }_{X}}$, which we denote by $\widetilde{\operatorname{Def}}_{X}$, is called the functor of generalized deformations of $X$; see [2]. We have shown in Section 2 that there exists a natural isomorphism $\psi: \operatorname{Def}_{\chi} \rightarrow \operatorname{Aut}_{H^{*}}(X, \mathbb{C})$. By these considerations, we obtain:

Theorem 4.4. The linear map

$$
\operatorname{Poly}_{X} \rightarrow \widetilde{C}(\chi), \quad \xi \mapsto\left(l_{\xi}, i_{\xi}\right),
$$


is a linear $L_{\infty}$-morphism and induces a natural transformation of functors

$$
\Phi: \widetilde{\operatorname{Def}}_{X} \rightarrow \operatorname{Aut}_{H *}(X, \mathbb{C})
$$

given at the level of Maurer-Cartan functors by the map $\xi \mapsto \psi_{\boldsymbol{i}_{\xi}}$.

Proposition 4.5. Via the natural identifications $H^{1}\left(\operatorname{Poly}_{X}\right)=\bigoplus_{i \geq 0} H^{i}\left(\wedge^{i} T_{X}\right)$ and $H^{*}(X, \mathbb{C})=\bigoplus_{p, q} H^{q}\left(X, \Omega_{X}^{p}\right)$ given by the Dolbeault's theorem and the $\partial \bar{\partial}$-lemma, the differential of $\Phi$,

$$
d \Phi: H^{1}\left(\operatorname{Poly}_{X}\right) \rightarrow \operatorname{Hom}^{0}\left(H^{*}(X, \mathbb{C}), H^{*}(X, \mathbb{C})\right),
$$

is identified with the contraction

$$
\left(\bigoplus_{i \geq 0} H^{i}\left(\wedge^{i} T_{X}\right)\right) \otimes\left(\bigoplus_{p, q} H^{q}\left(X, \Omega_{X}^{p}\right)\right) \rightarrow \bigoplus_{i, p, q} H^{q+i}\left(X, \Omega_{X}^{p-i}\right) .
$$

Proof. By Lemma 4.1 we have a commutative diagram of differential complexes

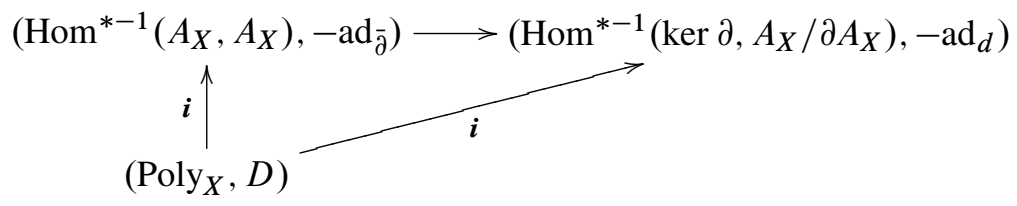

where we have used the fact that on ker $\partial$ and on $A_{X} / \partial A_{X}$ the differentials ad $d$ and ad $\bar{\partial}$ coincide. Using the identification $H_{\bar{\partial}}^{*}\left(A_{X}\right)=H^{*}(X, \mathbb{C})$ coming from Dolbeault's theorem and the $\partial \bar{\partial}$-lemma, and by Remark 2.1 , the above commutative diagram induces the commutative diagram in cohomology

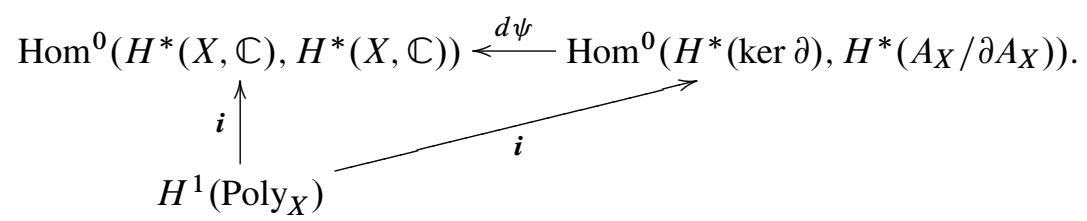

Since, by Theorem 4.4, the differential of $\Phi$ is $d \Phi=d \psi \circ \boldsymbol{i}$, which completes the proof.

As a corollary of Theorem 4.4 , the linear map $\xi \mapsto\left(\boldsymbol{l}_{\xi}, \boldsymbol{i}_{\xi}\right)$ induces a morphism of obstruction spaces $H^{2}$ (Poly) $\rightarrow \operatorname{Hom}^{1}\left(H^{*}(X, \mathbb{C}), H^{*}(X, \mathbb{C})\right)$ commuting with $\Phi$ and obstruction maps [5], [17]. The same argument of Proposition 4.5 shows that this morphism is naturally identified with the contraction

$$
\left(\bigoplus_{i \geq 0} H^{i+1}\left(\bigwedge^{i} T_{X}\right)\right) \otimes\left(\bigoplus_{p, q} H^{q}\left(X, \Omega_{X}^{p}\right)\right) \rightarrow \bigoplus_{i, p, q} H^{q+i+1}\left(X, \Omega_{X}^{p-i}\right) .
$$

Since the deformation functor $\operatorname{Aut}_{H *}(X, \mathbb{C})$ is smooth, we obtain the following version of the so-called Kodaira principle (ambient cohomology annihilates obstruction): 
Proposition 4.6. The obstructions to extended deformations of a compact Kähler manifold $X$ are contained in the subspace

$$
\bigoplus_{i \geq 0} \bigcap_{p, q} \operatorname{ker}\left(H^{i+1}\left(\bigwedge^{i} T_{X}\right) \stackrel{i}{\rightarrow} \operatorname{Hom}\left(H^{q}\left(X, \Omega_{X}^{p}\right), H^{q+i+1}\left(X, \Omega_{X}^{p-i}\right)\right)\right.
$$

of $H^{2}\left(\operatorname{Poly}_{X}\right)$.

As an immediate corollary we recover the fact that extended deformations of compact Calabi-Yau manifolds are unobstructed [2]. Indeed, if $X$ is an $n$-dimensional compact Calabi-Yau manifold, then the contraction pairing

$$
H^{i+1}\left(\bigwedge^{i} T_{X}\right) \otimes H^{0}\left(X, \Omega_{X}^{n}\right) \rightarrow H^{i+1}\left(X, \Omega_{X}^{n-i}\right)
$$

is nondegenerate for any $i \geq 0$.

\section{Restriction to classical deformations}

Let $\left(X, \mathcal{O}_{X}\right)$ be a complex manifold. It is well known that the infinitesimal deformations of the complex structure of $X$ are governed by the Kodaira-Spencer DGLA of $X$. More precisely, there is a natural isomorphism of deformation functors

$$
\operatorname{Def}_{K_{X}} \stackrel{\sim}{\rightarrow} \operatorname{Def}_{X}
$$

which map a Maurer-Cartan element $\xi \in A_{X}^{0,1}\left(T_{X}\right)$ to the complex manifold $\left(X, \mathcal{O}_{\xi}\right)$, where the structure sheaf $\mathcal{O}_{\xi}$ is defined by

$$
\mathcal{O}_{\xi}=\operatorname{ker}\left\{\bar{\partial}_{\xi} \mid \mathcal{A}_{X}^{0,0} \rightarrow \mathcal{A}_{X}^{0,1}\right\}=\left\{f \in \mathcal{A}_{X}^{0} \mid\left(\bar{\partial}+l_{\xi}\right) f=0\right\}
$$

see [4], [9], [14], Ex. 3.4.1, or [13]. The above equations must be interpreted as identities among functors of Artin rings; namely, they mean that for any local Artin algebra $\left(B, \mathfrak{m}_{B}\right)$ the Kuranishi data $\xi \in \operatorname{MC}_{\mathrm{KS}_{X}}(B) \subseteq A_{X}^{0,1}\left(T_{X}\right) \otimes \mathfrak{m}_{B}$ are mapped to the family $\left(X, \mathcal{O}_{\xi}\right)$ of complex manifolds over $\operatorname{Spec}(B)$, whose structure sheaf $\mathcal{O}_{\xi}$ is defined by

$$
\mathcal{O}_{\xi}=\operatorname{ker}\left\{\bar{\partial}_{\xi}: \mathcal{A}_{X}^{0,0} \otimes B \rightarrow \mathcal{A}_{X}^{0,1} \otimes B\right\}=\left\{f \in \mathcal{A}_{X}^{0} \otimes B \mid\left(\bar{\partial}+\boldsymbol{l}_{\xi}\right) f=0\right\} .
$$

Let now

$$
A_{X}=F_{\xi}^{0} \supseteq F_{\xi}^{1} \supseteq \cdots
$$

be the Hodge filtration of differential forms on the complex manifold $\left(X, \mathcal{O}_{\xi}\right)$, i.e., for every $m \geq 0, F_{\xi}^{m}$ is the complex of global sections of the differential ideal sheaf $\widetilde{F}_{\xi}^{m} \subseteq \mathcal{A}_{X}$ generated by $\left(d \mathcal{O}_{\xi}\right)^{m}$. Again, here we write $\mathcal{A}_{X}$ for the functor of Artin 
rings defined by $B \mapsto \mathcal{A}_{X} \otimes B$. If $X$ is a compact Kähler manifold, the cohomology of $\left(F_{\xi}^{m}, d\right)$ naturally embeds into the cohomology of $\left(A_{X}, d\right)$. Since the dimension of $H^{*}\left(F_{\xi}^{m}, d\right)$ is independent of $\xi$, one can look at $H^{*}\left(F_{\xi}^{m}, d\right)$ as a different linear embedding of $H^{*}\left(F^{m}, d\right)$ into $H^{*}(X ; \mathbb{C})$. Hence $\xi \mapsto H^{*}\left(F_{\xi}^{m}, d\right)$ is a map

$$
\operatorname{Def}_{X} \rightarrow \operatorname{Grass}_{H^{*}}\left(F^{m}\right), H^{*}(X ; \mathbb{C}),
$$

called the $m$-th period map.

The inclusion of DGLAs $\mathrm{KS}_{X} \hookrightarrow$ Poly $_{X}$ induces an embedding of deformation functors $\operatorname{Def}_{X} \rightarrow \widetilde{\operatorname{Def}_{X}}$. Hence, the restriction of $\Phi$ to $\operatorname{Def}_{X}$ is a natural transformation

$$
\Phi: \operatorname{Def}_{X} \rightarrow \operatorname{Aut}_{H^{*}(X, \mathbb{C})}
$$

Theorem 5.1. For any $m \geq 0$, the map $\Phi: \operatorname{Def}_{X} \rightarrow \operatorname{Aut}_{H^{*}(X, \mathbb{C})}$ lifts the m-th

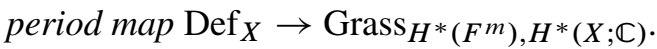

Proof. Let $\xi$ be a Maurer-Cartan element in $\mathrm{KS}_{X}$. Then $\left(\boldsymbol{l}_{\xi}, e^{\boldsymbol{i}_{\xi}}\right) \in \mathrm{MC}_{\chi}$ is a MaurerCartan element with $\boldsymbol{l}_{\xi}$ of bidegree $(0,1)$. Let $[\omega]$ be an element in $H^{*}\left(F^{m}\right)$. To compute $\psi_{\xi}[\omega]$ we pick a $\partial$-closed representative for the class $[\omega]$, which we can assume to be $\omega$, and then we take the cohomology class of a $d$-closed representative of $e^{\boldsymbol{i}_{\xi}} \omega$ in $e^{\boldsymbol{i}_{\xi}}(\operatorname{ker} \partial) / e^{\boldsymbol{i}_{\xi}}\left(\partial A_{X}\right)$, i.e., we have $\psi_{\xi}[\omega]=\left[e^{\boldsymbol{i}_{\xi}}(\omega-\partial \beta)\right]$ for any $\beta \in A_{X}$ such that $d e^{i_{\xi}}(\omega-\partial \beta)=0$. For such a $\beta$ we have

$$
0=e^{-i_{\xi}} d e^{i_{\xi}}(\omega-\partial \beta)=-\bar{\partial} \partial \beta+\boldsymbol{l}_{\xi}(\omega)-\boldsymbol{l}_{\xi}(\partial \beta)
$$

and so

$$
\left(\bar{\partial}+\boldsymbol{l}_{\xi}\right) \partial \beta=\boldsymbol{l}_{\xi}(\omega) .
$$

Write $\eta_{<m}$ and $\eta_{\geq m}$ for the components of a differential form $\eta$ in $\bigoplus_{i<m} A_{X}^{i, *}$ and in $\bigoplus_{i \geq m} A_{X}^{i, *}$, respectively. Since both $\boldsymbol{l}_{\xi}$ and $\left(\bar{\partial}+\boldsymbol{l}_{\xi}\right)$ are homogeneous of bidegree $(0,1)$, we have

$$
\boldsymbol{l}_{\xi}(\omega)=\left(\boldsymbol{l}_{\xi}(\omega)\right)_{\geq m}=\left(\left(\bar{\partial}+\boldsymbol{l}_{\xi}\right) \partial \beta\right)_{\geq m}=\left(\bar{\partial}+\boldsymbol{l}_{\xi}\right) \partial\left(\beta_{\geq m-1}\right) .
$$

Hence $\psi_{\boldsymbol{i}_{\xi}}[\omega]=\left[e^{\boldsymbol{i}_{\xi}}\left(\omega-\partial\left(\beta_{\geq m-1}\right)\right)\right] \in H^{*}\left(e^{\boldsymbol{i}_{\xi}} F^{m}\right)$, and so

$$
\Phi_{\xi}\left(H^{*}\left(F^{m}\right)\right)=H^{*}\left(e^{i_{\xi}} F^{m}\right) .
$$

On the other hand, the period of the infinitesimal deformation $\mathcal{O}_{\xi}=\operatorname{ker}\left(\bar{\partial}+\boldsymbol{l}_{\xi}\right)$ is $H^{*}\left(F_{\xi}^{m}\right) \subseteq H^{*}\left(A_{X}\right)$, where $F_{\xi}^{m}$ is the complex of global sections of the differential ideal sheaf $\mathscr{F}_{\xi}^{m} \subseteq \mathcal{A}_{X}$ generated by $\left(d \mathcal{O}_{\xi}\right)^{m}$. Since $e^{\boldsymbol{i}_{\xi}}$ is the identity on $\mathcal{A}_{X}^{0,0}$, by Lemma 2.2 we can write

$$
e^{-i_{\xi}}\left(d \mathcal{O}_{\xi}\right)=e^{-i_{\xi}} d e^{i_{\xi}} \mathcal{O}_{\xi}=\left(\partial+\bar{\partial}+\boldsymbol{l}_{\xi}\right) \mathcal{O}_{\xi}=\partial \mathcal{O}_{\xi} \subseteq \partial \mathcal{A}_{X}^{0,0} \subseteq \mathcal{A}_{X}^{1,0} .
$$


Since $e^{i_{\xi}}: \mathcal{A}_{X} \rightarrow \mathcal{A}_{X}$ is a morphism of sheaves of differential graded commutative

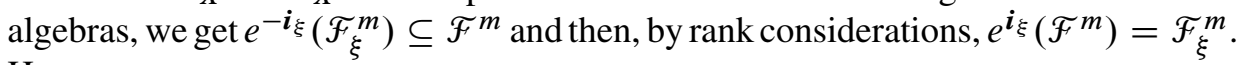
Hence

$$
\Phi_{\xi}\left(H^{*}\left(F^{m}\right)\right)=H^{*}\left(\mathscr{F}_{\xi}^{m}\right) .
$$

Remark 5.2. That the $m$-th period map $\operatorname{Def}_{X} \rightarrow \operatorname{Grass}_{H^{*}\left(F^{m}\right), H^{*}(X ; \mathbb{C})}$ is induced by an $L_{\infty}$-morphism was shown in [7]. Namely, let $\chi_{m}: L_{m} \hookrightarrow \operatorname{Hom}^{*}\left(A_{X}, A_{X}\right)$ the inclusion of the subalgebra

$$
L_{m}=\left\{f \in \operatorname{Hom}^{*}\left(A_{X}, A_{X}\right) \mid f\left(F^{m}\right) \subseteq F^{m}\right\}
$$

in the DGLA of endomorphisms of $A_{X}$. Then $\operatorname{Def}_{\chi_{m}} \simeq \operatorname{Grass}_{H^{*}\left(F^{m}\right), H^{*}(X)}$, and the map $\xi \mapsto\left(\boldsymbol{l}_{\xi}, \boldsymbol{i}_{\xi}\right)$ is an $L_{\infty}$-morphism between $\mathrm{KS}_{X}$ and $C_{\chi_{m}}$ inducing the $m$-th period map.

\section{References}

[1] R. Abraham and J. E. Marsden, Foundations of mechanics. 2nd ed., Benjamin/Cummings Publishing Co. Inc. Advanced Book Program, Reading, Mass., 1978. Zbl 0393.70001 MR 0515141

[2] S. Barannikov and M. Kontsevich, Frobenius manifolds and formality of Lie algebras of polyvector fields. Internat. Math. Res. Notices 1998 (1998), 201-215. Zbl 0914.58004 MR 1609624

[3] M. Bershadsky, S. Cecotti, H. Ooguri, and C. Vafa, Kodaira-Spencer theory of gravity and exact results for quantum string amplitudes. Comm. Math. Phys. 165 (1994), 311-427. Zbl 0815.53082 MR 1301851

[4] H. Clemens, Geometry of formal Kuranishi theory. Adv. Math. 198 (2005), 311-365. Zbl 1092.14004 MR 2183257

[5] B. Fantechi and M. Manetti, Obstruction calculus for functors of Artin rings, I. J. Algebra 202 (1998), 541-576. Zbl 0981.13009 MR 1617687

[6] D. Fiorenza and M. Manetti, $L_{\infty}$ structures on mapping cones. Algebra Number Theory 1 (2007), 301-330. Zbl 1166.17010 MR 2361936

[7] D. Fiorenza and M. Manetti: $L_{\infty}$ algebras, Cartan homotopies and period maps. Preprint 2006. arXiv:math/0605297.

[8] K. Fukaya, Deformation theory, homological algebra and mirror symmetry. In Geometry and physics of branes (Como, 2001), Ser. High Energy Phys. Cosmol. Gravit., IOP, Bristol 2003, 121-209. MR 1950958, http://www.math.kyoto-u.ac.jp/ fukaya/fukaya.html

[9] W. M. Goldman and J. J. Millson, The homotopy invariance of the Kuranishi space. Illinois J. Math. 34 (1990), 337-367. Zbl 0707.32004 MR 1046568

[10] M. L. Green, Infinitesimal methods in Hodge theory. In Algebraic cycles and Hodge theory (Torino, 1993), Lecture Notes in Math. 1594, Springer, Berlin 1994, 1-92. Zbl 0846.14001 MR 1335239 
[11] P. A. Griffiths, Periods of integrals on algebraic manifolds. II. Local study of the period mapping. Amer. J. Math. 90 (1968), 805-865. Zbl 0183.25501 MR 0233825

[12] M. Gualtieri, Generalized geometry and the Hodge decomposition. Oberwolfach Rep. 1 (2004), no. 38, 2015-2019.

[13] D. Iacono, Differential graded lie algebras and deformations of holomorphic maps. PhD Thesis, Università degli Studi di Roma "La Sapienza", Roma 2006. arXiv:math.AG/0701091

[14] M. Kontsevich, Deformation quantization of Poisson manifolds. Lett. Math. Phys. 66 (2003), 157-216. Zbl 1058.53065 MR 2062626

[15] T. Lada and M. Markl, Strongly homotopy Lie algebras. Comm. Algebra 23 (1995), 2147-2161. Zbl 0999.17019 MR 1327129

[16] T. Lada and J. Stasheff, Introduction to sh Lie algebras for physicists. Internat. J. Theoret. Phys. 32 (1993), 1087-1103. Zbl 0824.17024 MR 1235010

[17] M. Manetti, Extended deformation functors. Internat. Math. Res. Notices 2002 (2002), 719-756. Zbl 1063.58007 MR 1891232

[18] M. Manetti, Cohomological constraint on deformations of compact Kähler manifolds. Adv. Math. 186 (2004), 125-142. Zbl 1075.53074 MR 2065509

[19] M. Manetti, Lectures on deformations of complex manifolds (deformations from differential graded viewpoint). Rend. Mat. Appl. (7) 24 (2004), 1-183. Zbl 1066.58010 MR 2130146

[20] M. Manetti, Lie description of higher obstructions to deforming submanifolds. Ann. Sc. Norm. Super. Pisa Cl. Sci. (5) 6 (2007), 631-659. Zbl 05292692 MR 2394413

[21] C. Voisin, Théorie de Hodge et géométrie algébrique complexe. Cours Spécialisés 10, Société Mathématique de France, Paris 2002. Zbl 1032.14001 MR 1988456

Received October 21, 2008; revised January 8, 2009

D. Fiorenza, M. Manetti, Dipartimento di Matematica “Guido Castelnuovo”, Università di Roma "La Sapienza”, P.le Aldo Moro 5, 00185 Roma, Italy

E-mail: fiorenza@mat.uniroma1.it,manetti@mat.uniroma1.it 